\title{
Schutz vor HIV - aber nur bei guter Adhärenz!
}

\author{
Die „Pille gegen Aids“ wirkt; das haben mittlerweile mehrere Studien bestätigt. In der Praxis \\ scheitert das Konzept jedoch oft an mangeInder Therapietreue. Abhilfe schafft möglicherweise \\ ein intermittierendes Regime oder in Zukunft auch die Darreichung in Spritzenform.
}

\begin{abstract}
Mit einer oralen Präexpositionsprophylaxe (PrEP) auf der Basis von Tenofovir lässt sich eine HIV-Infektion mit hoher Wahrscheinlichkeit verhindern; das hat die iPrEX-Studie im Jahr 2010 gezeigt. Therapietreue ist dabei äußerst wichtig, wie die Erweiterungsstudie, iPrEX OLE (http://iprexole.com/) bestätigt. Hier sank das Ansteckungsrisiko bei guter Adhärenz um $84 \%$.
\end{abstract}

\section{„PrEP bei Bedarf“ in Europäischen Leitlinien verankert}

Das derzeit einzige verfügbare Präparat für die PrEP - eine Kombination aus Emtricitabin und Tenofovir (FTC/TDF, Truvada $^{\circledR}$ ) hat hierzulande zwar noch keine Marktzulassung (diese wird bis Ende 2016 erwartet); es kann aber z. B. in HIV-Schwerpunktpraxen auf Privatrezept verschrieben werden. Nach den Leitlinien der EACS (European AIDS Clinical Society) besteht für die Anwendung die Wahl zwischen zwei Optionen:

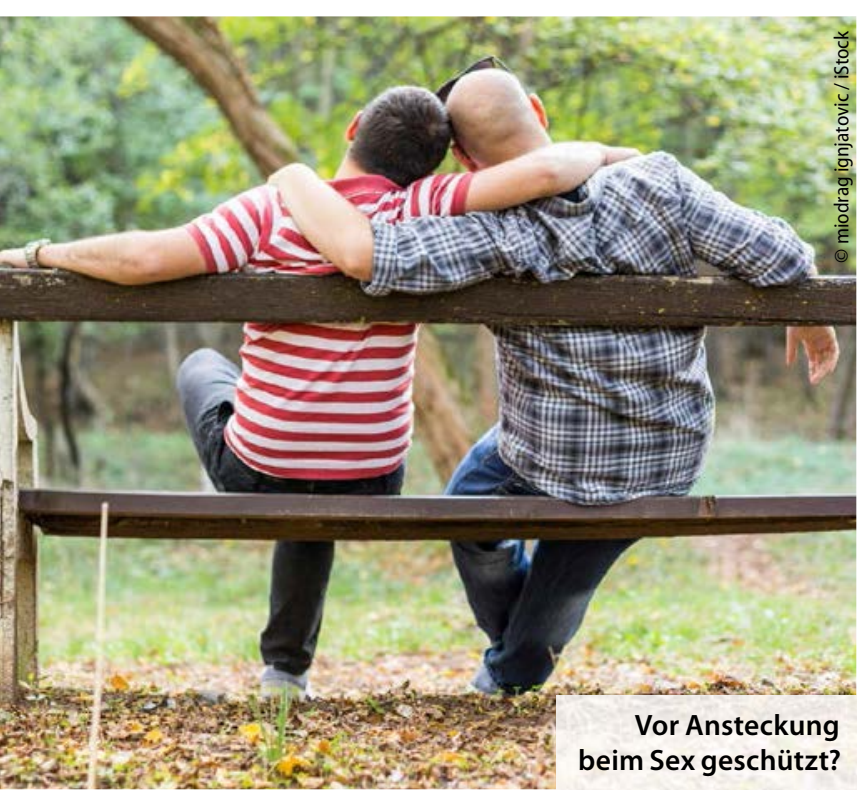

Die Tabletten können täglich oder „bei Bedarf“ in Form einer sog. intermittierenden Therapie eingenommen werden.

Das Bedarfsregime ist allerdings nur bei Männern ausreichend wirksam (nur in der Rektal-, nicht in der Scheidenschleimhaut werden ausreichend hohe Konzentrationen erreicht). Die „PrEP on demand“ beruht auf der französischen IPERGAY-Studie: Dort wurde gezeigt, dass es offenbar genügt, mit einer doppelten Dosis innerhalb von 2 bis 24 Stunden vor dem geplanten Sex zu beginnen und dann an zwei darauffolgenden Tagen jeweils noch eine Einzeldosis zu nehmen.

Insgesamt sollte eine Höchstdosis von sieben Tabletten pro Woche nicht überschritten werden. Langfristig sind regelmäßig die Nierenfunktion und Knochendichte zu überprüfen.

\section{3-Monats-Spritze funktioniert nicht} Um Problemen mit der Adhärenz zu begegnen, werden derzeit weitere Strategien erprobt. Auf der CROI* wurden die Ergebnisse der ECLAIR-Studie vorgestellt. Hier hatte man die PrEP in Spritzenform alle 3 Monate i.m. verabreicht. Ermöglicht wird dies - zumindest theoretisch - durch Cabotegravir LA, eine sog. Long Acting Nanosuspension. In einer Phase-2a-Studie mit 127 Männern war die Substanz gut verträglich, allerdings haperte es mit der Effektivität: Bei bis zu 31\% der Visiten fanden sich bei den Männern im Verumarm subtherapeutische Spiegel, in einem Fall kam es trotz Therapie zu einer Serokonversion nach
HIV-Exposition. Wie Dr. Stefan Scholten, Köln, resümierte, sind mit einem Dreimonatsintervall offenbar keine ausreichenden Plasmaspiegel zu erzielen. Die folgenden Studien werden nun für ein Intervall von acht Wochen aufgesetzt.

Außerdem in Entwicklung: ein Rektalgel auf Basis von Tenofovir sowie die Kombination FTC/TAF. TAF steht für Tenofoviralafenamid. Die Substanz ist gezielt auf die Lymphozyten gerichtet. Man erhofft sich weniger Nebenwirkungen. Dr. Elke Oberhofer

*C Markowitz M et al, \#106; CROI 2016.

- Münchner AIDS- und Hepatitis-Tage, 11.-13. März 2016

\section{PrEP für Frauen: Was bringt der Vaginalring?}

Die Wirksamkeit einer HIV-Präexpositionsprophylaxe mithilfe eines Dapivirin-haltigen Vaginalrings wurde in zwei Studien mit afrikanischen Frauen überprüft. An der ASPIRE-Studie nahmen 2.629 Frauen teil, an der RING-Studie 1.959 Frauen. In beiden Studien war die Schutzrate mit 27\% bzw. 31\% im jeweiligen Gesamtkollektiv enttäuschend niedrig; dies lag den $\mathrm{Au}$ toren zufolge vor allem an der schlechten Adhärenz. Wie Dr. Gaby Knecht vom Infektiologikum in Frankfurt berichtete, war der Ring in beiden Studien Bestandteil eines Präventionspakets: Die Frauen waren zu Safer-Sex-Praktiken (inkl. Kondom) beraten worden; zusätzlich hatte man innen den Vaginalring zur Verfügung gestellt und gezeigt, wie dieser eingesetzt wird. Letzterer sollte alle vier Wochen von der Teilnehmerin selbst gewechselt werden. Dass dies schlecht funktionierte, zeigten die im Ring verbliebenen Wirkstoffkonzentrationen. Insbesondere junge Frauen unter 22 Jahren hatten den Vaginalring kaum benutzt. „Diese jungen Menschen müssen wir mit anderen Präventionsprogrammen erreichen", sagte Knecht. = eo

- Baeten J et al, \#109 LB; Nel A et al, \#110 LB; CROI 2016 